\title{
Ventilatory Responses to Whole Body Cryotherapy
}

Dr Lucy Hammond ${ }^{\mathrm{a} *}$, Miss Katie Mitchell ${ }^{\mathrm{b}, \mathrm{c}}$, Mr Saul Cuttell $^{\mathrm{d}}$

*Corresponding author

${ }^{a}$ Warwick Medical School, University of Warwick, Coventry, CV4 7AL, UK;

1.hammond.1@warwick.ac.uk

bBirmingham City University, 15 Bartholomew Road, Birmingham, B5 5JU, UK;

katie.mitchell@bcu.ac.uk

${ }^{c}$ Moulton College, West Street, Moulton, Northamptonshire, NN3 7RR, UK;

${ }^{\mathrm{d}}$ University of Northampton, University Drive, Northampton, NN1 5PH, UK;

Saul.Cuttell@northampton.ac.uk

\section{Declarations of interest: none}

Funding: This research did not receive any specific grant from funding agencies in the public, commercial, or not-for-profit sectors.

\begin{abstract}
The effects of whole body cryotherapy (WBC) on measures of respiratory function, and their interaction with sex and anthropometry, are unknown. This study investigated ventilatory responses after WBC and examined the role of sex and anthropometric characteristics in 22 recreationally active male and female participants. Thermal sensation [TS], heart rate, blood pressure and ventilatory measures (expired volume [ $\dot{\mathrm{V} E}$ ], oxygen consumption [ $\dot{\mathrm{V}}_{2}$ ], respiratory exchange ratio and metabolic equivalents of task [METS]) were evaluated preand post-WBC, compared to a control condition. Vं E increased by 10.8 L.min ${ }^{-1}$ following WBC compared to $0.1 \mathrm{~L} \mathrm{~min}^{-1}$ in the control session ( $p=.001$ ). $\dot{\mathrm{V}} \mathrm{O}_{2}$ increased by $0.4 \mathrm{~L}_{\mathrm{min}} \mathrm{m}^{-1}$ following WBC compared to 0.0 L. $\mathrm{min}^{-1}$ in the control session $(p<.001)$. METS increased by 1.0 following WBC compared to 0.1 in the control session $(p<.001)$. TS decreased by 2 following WBC compared to 0 in the control session ( $p<.001)$, with females' TS decreasing significantly more than males following WBC ( $p=.001)$. Following a WBC exposure, highly significant strong negative relationships were observed between fat mass index and $\Delta \dot{\mathrm{V}} \mathrm{O}_{2}$ $(p=.002, r=-.718)$, body fat percentage and $\Delta \dot{\mathrm{V}}_{2}(p=.003, r=-.700)$, fat mass index and $\Delta$ METS $(p=.001, r=-.729)$, body fat percentage and $\triangle$ METS $(p=.004, r=-.676)$.
\end{abstract}

Furthermore, strong, statistically significant positive correlations were observed following a 
WBC exposure between BSA to mass ratio and TS $(p=.005, r=-.669)$, TS and BSA $(p<0.001$, $r=.776)$ and fat free mass index and TS ( $p=.001, r=.745)$. A short exposure to WBC elicits a ventilatory response via increased $\dot{\mathrm{V}} \mathrm{O}_{2}, \dot{\mathrm{V}} \mathrm{E}$ and METS. The largest increases occur within the first minute post-exposure, but return to near resting levels one minute post-WBC. Increases in $\dot{\mathrm{V}} \mathrm{O}_{2}$, $\dot{\mathrm{V}} \mathrm{E}$ and METS observed post-WBC are likely due to an acute transient shivering response due to cold air exposure.

Key words: Thermoregulation, Sexual dimorphism, Anthropometry, Temperature, Cold exposure, Shivering 


\subsection{Introduction}

Whole body cryotherapy (WBC) involves brief exposures, usually of up to 3 minutes, in freezing temperatures often around $-140^{\circ} \mathrm{C}$ (Selfe et al., 2014). It was first used in the treatment of rheumatoid arthritis (Yamauchi, 1986) and has become a popular treatment for ankylosing spondylitis (Stanek et al., 2018), fibromyalgia, osteoporosis and multiple sclerosis (Cholewka et al., 2012), depressive and anxiety disorders (Rymaszewska et al., 2008) and as a recovery tool for sports teams and athletes (Lombardi et al., 2017). Thus far, minimal adverse effects have been reported from treatment with WBC, suggesting it is a safe cryotherapy treatment suitable for healthy individuals (Costello et al., 2015). Although there is an established field of research in cold water immersion concerned with cooling and rewarming, and potential metabolic effects of cold, there are comparatively little data available in this field when concerned with the use of WBC. While its efficacy for athletic recovery remains unknown, there is a growing body of evidence that shows that differences in cooling responses exist between males and females and between individuals with different morphology, with fatter participants' skin cooling more quickly than leaner participants (Cholewka et al., 2012; Hammond et al., 2014; Cuttell et al., 2017, Bouzigon et al., 2018).

In extremely cold conditions, the classical cold shock response is characterised by a pattern of reflexes driven by cutaneous thermoreceptors which results in tachycardia, a respiratory gasp, uncontrollable hyperventilation, peripheral vasoconstriction and hypertension (Tipton, 1989; Datta and Tipton, 2006). The cold shock response can have obvious negative outcomes when coupled with cold water immersion (i.e. hypothermia and death), yet little is known of the cold shock and respiratory response to WBC. There are conflicting reports on the effects of WBC on ventilatory measures and lung function, with Engel et al. (1989) reporting a transient bronchodilatory effect whereas Smolander et al. (2006) reported minor bronchoconstriction 30 minutes post-WBC. In the case of metabolism, cold exposure leads to increased heat production to maintain equilibrium, with cold-induced thermoregulation associated with an increase in lipid metabolism (Vallerand \& Jacobs, 1989). Ten cryotherapy sessions have been found to reduce serum trigylcerides in active males, with 20 sessions resulting in reduced total cholesterol, low density lipoproteins and triglycerides and increased high density lipoproteins (Lubowska et al., 2010). Similarly, ten daily whole-body cryotherapy sessions coupled with 60 minutes of kinesiotherapy significantly decreased low density lipoproteins, decreased oxidative stress markers and increased total antioxidant 
capacity compared to kinesiotherapy alone (Stanek et al., 2019). These studies provide insight into physiological responses to WBC, however effects of WBC on broader measures of respiratory function, and their interaction of with sex and anthropometry, remain unknown in both healthy subjects and patients. Considering the metabolic and respiratory effects of WBC would be useful for furthering understanding of how athletes respond to such treatments, the potential of use for health-related conditions such as asthma, and even the physiological reactions to such an extreme environment (for example, polar expeditions).

This study aims to investigate the respiratory response and energy expenditure after WBC, and to examine the role of sexual dimorphism and anthropometric characteristics in relation to the shivering response and energy expenditure. A secondary aim of the study was to explore the feasibility of collecting metabolic data following WBC using different methods.

\subsection{Materials and Methods}

Twenty-two recreationally active participants (females: $n=8$, males: $n=14$ ) gave written informed consent to participate in the study, which was approved by Moulton College Ethics Committee in accordance with the Declaration of Helsinki. Participants completed a medical form and declared they were free from chronic or cold sensitive diseases such as Raynaud's disease, cold urticaria, asthma, claustrophobia, cardiac or respiratory conditions. This study comprised two phases; in phase 1 , the main study, males $(n=9)$ and females $(n=8)$ shivering responses to whole body cryotherapy were evaluated using Douglas bag methodology. Phase 2 comprised a smaller inquiry of a separate group of males $(n=5)$ to investigate shivering responses to the same exposure using breath-by-breath analysis. A summary of anthropometric characteristics of the participants can be seen in Table 1. 


\begin{tabular}{|c|c|c|c|c|c|c|c|}
\hline & $\begin{array}{l}\text { PHAS } \\
\text { Males } \\
\text { Mean }\end{array}$ & SD & $\begin{array}{l}\text { Female } \\
\text { Mean }\end{array}$ & SD & $\begin{array}{l}P \\
\text { value } \\
\end{array}$ & $\begin{array}{l}\text { PHASI } \\
\text { Males } \\
\text { Mean }\end{array}$ & SD \\
\hline Age (years) & 23.1 & 3.4 & 26.0 & 7.0 & & 28.4 & 3.9 \\
\hline Height $(\mathrm{cm})$ & 178.0 & 5.9 & 168.0 & 7.0 & * & 182.0 & 5.5 \\
\hline Mass (kg) & 81.9 & 8.5 & 66.6 & 9.6 & $\beta$ & 81.2 & 10.8 \\
\hline Body Mass Index $\left(\mathrm{kg} / \mathrm{m}^{2}\right)$ & 25.9 & 2.3 & 23.3 & 2.7 & $*$ & 24.5 & 4.0 \\
\hline Body Surface Area $\left(\mathrm{m}^{2}\right)$ & 2.0 & 0.12 & 1.76 & 0.15 & $\beta$ & 2.02 & 0.1 \\
\hline BSA to Mass Ratio $\left(\mathrm{cm}^{2} / \mathrm{kg}\right)$ & 2.45 & 0.12 & 2.6 & 0.21 & * & 2.51 & 0.22 \\
\hline Fat Mass Index $\left(\mathrm{kg} / \mathrm{m}^{2}\right)$ & 2.83 & 1.25 & 5.83 & 1.58 & $\beta$ & - & - \\
\hline Fat Free Mass Index $\left(\mathrm{kg} / \mathrm{m}^{2}\right)$ & 23.11 & 2.14 & 17.53 & 1.53 & $\beta$ & - & - \\
\hline Body fat percentage (BIA) & 10.8 & 4.5 & 24.7 & 4.3 & $\beta$ & - & - \\
\hline Body fat percentage (skinfold) & 11.1 & 2.2 & 22.8 & 8.0 & $\beta$ & - & - \\
\hline Chest skinfold (mm) & 3.8 & 1.3 & 11.1 & 4.7 & $\beta$ & - & - \\
\hline Axilla skinfold (mm) & 6.2 & 2.9 & 16.8 & 9.5 & $\beta$ & - & - \\
\hline Tricep skinfold (mm) & 6.2 & 1.3 & 21.4 & 11.6 & $\beta$ & - & - \\
\hline Subscapular skinfold (mm) & 8.7 & 1.5 & 14.8 & 5.7 & $\beta$ & - & - \\
\hline Abdominal skinfold (mm) & 10.4 & 4.7 & 15.0 & 10.0 & & - & - \\
\hline Suprailiac skinfold (mm) & 4.9 & 2.0 & 12.4 & 5.9 & $\beta$ & - & - \\
\hline Thigh skinfold (mm) & 6.3 & 2.7 & 27.4 & 15.3 & $\beta$ & - & - \\
\hline
\end{tabular}

\subsection{Instrumentation and measures}

Height and mass were measured using a standard stadiometer (SECA 213, UK) and weighing scales (SECA Scales, UK). Body composition was measured using bioelectrical impedance (BIA) at a frequency of $50 \mathrm{kHz}$ (Biostat 1500, Isle of Man), and skinfold calipers

(Harpenden) at seven sites (chest, axilla, tricep, subscapula, abdominal, suprailliac and thigh), with three measures taken at each site in order to establish a mean measurement. From the measures taken, body mass index (BMI), fat mass index (FMI), fat-free mass index (FFMI) and body surface area (BSA) using the DuBois equation (DuBois and DuBois, 1916) were calculated.

Cardiovascular measurements comprised blood pressure (BP) (Omron M6, Matasuaka Mie, Japan) and heart rate (HR) (Polar heart rate FT2, Finland). Thermal sensation (TS) was measured using a 9-point scale, which ranged from -4 (very cold) to 0 (neutral) to 4 (very hot) (Westerlund et al., 2003). During Phase 1 of the study, participants' ventilated air was 
measured using Douglas bags and a manufacturer calibrated Servomex (Servomex MiniFoodPack 5200, Netherlands) analysis to establish expired volume ( $\dot{V}$ E) and volumes of expired oxygen $\left(\mathrm{O}_{2}\right)$ and carbon dioxide $\left(\mathrm{CO}_{2}\right)$. In Phase 2, ventilated air was measured using a laboratory calibrated Cortex Metalyzer 3B Metabolic System (Cranlea). In both study phases, these measures were used to calculate oxygen consumption $\left(\dot{\mathrm{V} \mathrm{O}}_{2}\right)$, respiratory exchange ratio (RER) and metabolic equivalents of task (METS). In Phase 2, breathing frequency $(\mathrm{BF})$, fraction of carbon dioxide in expired air $\left(\mathrm{FECO}_{2}\right)$ and fraction of oxygen in inspired air $\left(\mathrm{FO}_{2}\right)$ were also recorded.

Cryotherapy exposures took place in a temperature controlled, liquid nitrogen cooled, whole body cryotherapy chamber (Juka, Poland), at The Chris Moody Sports Injury and Rehabilitation Centre in Northamptonshire, United Kingdom. This comprised two chambers $\left(-60^{\circ} \mathrm{C}\right.$ and $\left.-140^{\circ} \mathrm{C}\right)$, connected by an internal door.

\subsection{Procedure}

\subsubsection{Phase 1}

Seventeen participants (males: $n=9$; females: $n=8$ ) attended the laboratory wearing shorts (males) or shorts and vest (females). All participants were instructed to refrain from consuming caffeine, smoking or taking part in exercise on the testing days and were advised to remain hydrated and to not consume food in the two hours prior to testing as substrate utilisation has been suggested to influence the shivering response (Tipton et al., 1997).

Height, mass, skinfold measures, HR and BP were initially measured. Participants then lay flat on a plinth for 20 minutes to acclimate to ambient room temperature. Four-electrode BIA was undertaken and connected at two sites (wrist/hand and ankle/foot). Participants then sat quietly to provide a three-minute resting Douglas bag sample. Half way through the sample collection, participants indicated their TS levels. They then walked for 30 seconds on a treadmill (Cybex Legacy 750T, Massachusetts USA) at $3 \mathrm{~km} \cdot \mathrm{h}^{-1}$, and then stood still for a three-minute resting period. This protocol was designed to replicate walking from the laboratory to the cryotherapy chamber and standing still for three minutes during the WBC exposure. Participants then immediately provided a further seated three-minute Douglas bag sample, with TS again taken after 1 minute 30 seconds. HR and BP measurements were also repeated. 
On the second presentation to the laboratory, participant's initial cardiovascular, metabolic and perceptual measures were taken in the same way as on the first occasion. Having removed jewellery, piercings and contact lenses, they then walked to the WBC chamber where they were provided with the appropriate safety garments (headband to cover the ears, surgical mask to cover the mouth, gloves, socks, clogs, tubular bandages for elbows and knees) and received a safety briefing by the chamber operators. Participants entered the antechamber $\left(-60^{\circ} \mathrm{C}\right)$ in pairs for thirty seconds, then transferred through the internal door into the main chamber $\left(-140^{\circ} \mathrm{C}\right)$, where they stayed for two minutes and thirty seconds. This exposure is similar to that used in previous research (Selfe et al., 2014) and is considered to be safe. Immediately upon exiting the chamber, participants sat for a further three-minute Douglas bag sample in the chamber lobby. HR, BP and TS measures were recorded again after 1 minute 30 seconds.

All participants completed the testing sequence in the same (non-randomised) order. Differences were present in the testing protocols experienced by males and females, where the male participants underwent all testing in the afternoon, with the control (pre- and posttest) and WBC (pre- and post-test) condition taking place one month apart. The female participants underwent all testing on the same day, with the control (pre- and post-test) condition taking place in the morning and WBC (pre- and post-test) condition taking place in the afternoon of the same day.

\subsubsection{Phase 2}

Five male participants attended the laboratory on one occasion, wearing shorts, and had height, mass and blood pressure measured. The participants were then fitted for a mask and sat quietly to provide a two-minute resting ventilatory sample. They completed the same WBC exposure as in Phase 1, and immediately upon exiting the chamber, provided a second two-minute resting ventilatory sample.

\subsection{Statistical Analysis}

All data are expressed as means and standard deviations, with the exception of TS, which is expressed as median and interquartile range. All statistical analyses were performed in SPSS (Statistical Package for the Social Sciences) version 26.0 (SPSS Inc, Chicago, IL). For Phase 1 of the study, independent t-tests were used to explore differences in anthropometric 
measures between males and females, with statistical significance accepted at $p \leq .05$. Delta $(\Delta)$ values were calculated for the control condition ( $\Delta$ control) and the WBC condition $(\triangle \mathrm{WBC})$ by subtracting pre- values from post- values. These change-data were analysed with non-parametric statistics, in order to determine whether there were differences between conditions and between sexes for heart rate, systolic and diastolic blood pressure, $\mathrm{O}_{2}, \mathrm{CO}_{2}, \dot{\mathrm{V}}$ E, $\dot{\mathrm{V}} \mathrm{O}_{2}$, METS, RER and TS. Wilcoxon Signed Rank Tests were undertaken for analyses between conditions and Mann-Whitney $U$ tests were undertaken for analyses between sex. Bonferroni adjustments were applied for multiple comparisons, to give an alpha level of $p \leq .017$. To explore whether relationships were present between anthropometric measures and $\triangle \mathrm{WBC}$ for ventilatory, metabolic and perceptual measures, Spearman rank correlations were conducted. Bonferroni adjustments were applied for multiple comparisons, to give an alpha level of $p \leq .008$. In Phase 2 , descriptive analyses (mean and standard deviation) were performed.

\subsection{Results}

\subsection{Phase 1}

Descriptive data for ventilatory, metabolic and cardiovascular measures are presented in Table 2. 
Table 2 Descriptive ventilatory, metabolic and cardiovascular data

\begin{tabular}{|c|c|c|c|c|c|c|c|c|c|c|c|}
\hline & \multicolumn{2}{|c|}{ Baseline } & \multicolumn{2}{|c|}{ Post-control } & \multirow{2}{*}{$\begin{array}{c}\Delta \text { control } \\
\text { Mean } \\
\end{array}$} & \multicolumn{2}{|c|}{ Pre-WBC } & \multicolumn{2}{|c|}{ Post-WBC } & \multirow{2}{*}{$\begin{array}{c}\Delta W B C \\
\text { Mean }\end{array}$} \\
\hline & & Mean & SD & Mean & SD & & Mean & SD & Mean & SD & \\
\hline \multicolumn{12}{|l|}{ Ventilatory and metabolic measures } \\
\hline \multirow[t]{3}{*}{ Expired oxygen (\%) } & males & 17.1 & 0.9 & 17.0 & 0.8 & -0.1 & 17.1 & 0.8 & 17.1 & 0.9 & -0.1 \\
\hline & females & 17.6 & 1.6 & 17.4 & 0.6 & -0.1 & 17.3 & 0.7 & 17.6 & 0.7 & 0.4 \\
\hline & combined & 17.2 & 0.8 & 17.2 & 0.7 & -0.1 & 17.2 & 0.7 & 17.4 & 0.8 & 0.2 \\
\hline \multirow[t]{3}{*}{ Expired carbon dioxide $(\%)$} & males & 3.2 & 0.5 & 3.1 & 0.8 & -0.1 & 3.1 & 0.7 & 3.0 & 0.8 & -0.1 \\
\hline & females & 2.8 & 0.5 & 2.9 & 0.7 & 0.2 & 2.8 & 0.5 & 2.9 & 0.4 & 0.0 \\
\hline & combined & 3.0 & 0.5 & 3.0 & 0.7 & 0.0 & 3.0 & 0.6 & 3.0 & 0.6 & 0.0 \\
\hline \multirow[t]{3}{*}{ METS } & males & 1.5 & 0.4 & 1.8 & 0.5 & 0.3 & 1.5 & 0.3 & 2.7 & 0.6 & 1.2 \\
\hline & females & 1.1 & 0.3 & 1.0 & 0.3 & -0.1 & 1.1 & 0.3 & 1.8 & 0.4 & 0.7 \\
\hline & combined & 1.2 & 0.4 & 1.4 & 0.6 & $0.1^{*}$ & 1.3 & 0.4 & 2.3 & 0.7 & $1.0^{\beta}$ \\
\hline \multirow[t]{3}{*}{ RER } & males & 0.8 & 0.1 & 0.7 & 0.1 & 0.0 & 0.8 & 0.1 & 0.7 & 0.1 & 0.0 \\
\hline & females & 0.8 & 0.1 & 0.8 & 0.1 & 0.0 & 0.7 & 0.1 & 0.8 & 0.1 & 0.1 \\
\hline & combined & 0.8 & 0.1 & 0.8 & 0.1 & 0.0 & 0.8 & 0.1 & 0.8 & 0.1 & 0.0 \\
\hline \multirow[t]{3}{*}{ Expired volume ( $\left.\dot{\mathrm{V}} \mathrm{E}, \mathrm{L} \cdot \mathrm{min}^{-1}\right)$} & males & 16.6 & 7.6 & 17.9 & 8.9 & 1.7 & 16.2 & 8.8 & 28.3 & 11.1 & 12.1 \\
\hline & females & 10.7 & 2.9 & 9.2 & 1.8 & -1.5 & 10.1 & 2.6 & 19.5 & 5.4 & 9.4 \\
\hline & combined & 13.7 & 6.7 & 13.8 & 8.1 & 0.1 & 13.1 & 7.0 & 23.9 & 9.6 & $10.8^{\beta}$ \\
\hline \multirow[t]{3}{*}{ Oxygen consumption $\left(\dot{\mathrm{V}} \mathrm{O}_{2}, \mathrm{~L} \cdot \mathrm{min}^{-1}\right)$} & males & 0.4 & 0.1 & 0.5 & 0.2 & 0.1 & 0.4 & 0.1 & 0.7 & 0.2 & 0.4 \\
\hline & females & 0.2 & 0.0 & 0.2 & 0.1 & 0.0 & 0.2 & 0.0 & 0.4 & 0.1 & 0.3 \\
\hline & combined & 0.3 & 0.1 & 0.4 & 0.2 & 0.0 & 0.3 & 0.1 & 0.6 & 0.2 & $0.4^{\beta}$ \\
\hline \multicolumn{12}{|l|}{ Cardiovascular measures } \\
\hline \multirow[t]{3}{*}{ Heart rate $(\mathrm{BPM})$} & males & 59.1 & 9.3 & 66.1 & 14.4 & 4.3 & 62.1 & 7.4 & 61.8 & 8.9 & -0.4 \\
\hline & females & 71.6 & 15.3 & 73.1 & 15.0 & 1.5 & 68.3 & 13.0 & 75.8 & 15.1 & 7.5 \\
\hline & combined & 65.4 & 14.0 & 68.3 & 14.3 & 2.9 & 65.2 & 10.7 & 68.8 & 14.0 & 3.6 \\
\hline \multirow[t]{3}{*}{ Systolic blood pressure $(\mathrm{mmHg})$} & males & 140.1 & 20.8 & 126.4 & 19.8 & -13.0 & 131.9 & 16.3 & 130.5 & 14.9 & -1.4 \\
\hline & females & 134.9 & 10.5 & 129.6 & 10.8 & -5.3 & 132.5 & 12.1 & 139.9 & 17.0 & 7.4 \\
\hline & combined & 139.0 & 16.1 & 130.0 & 14.1 & -9.1 & 132.1 & 135.2 & 135.2 & 16.2 & $3.0^{\S}$ \\
\hline \multirow[t]{3}{*}{ Diastolic blood pressure $(\mathrm{mmHg})$} & males & 67.2 & 8.7 & 72.1 & 11.3 & 5.0 & 72.0 & 8.6 & 71.9 & 8.0 & -0.1 \\
\hline & females & 80.6 & 11.8 & 80.0 & 8.4 & -0.6 & 79.6 & 11.4 & 77.9 & 11.9 & -1.8 \\
\hline & combined & 74.0 & 12.3 & 76.2 & 11.0 & 2.2 & 75.8 & 10.5 & 74.9 & 10.3 & -0.1 \\
\hline
\end{tabular}

* statistically significant difference between males and females, $p \leq .017$

$\beta$ statistically significant difference between $\Delta$ control and $\triangle \mathrm{WBC}, p \leq .001$

$\S$ statistically significant difference between $\Delta$ control and $\Delta \mathrm{WBC}, p=.006$ 


\subsubsection{Ventilatory and metabolic measures}

No significant differences between conditions or sex were observed for expired oxygen, expired carbon dioxide or RER. For V E, $\triangle \mathrm{WBC}$ was significantly greater than $\Delta$ control $(Z=$ $-3.309, p=.001$ ), with an increase of $10.8 \mathrm{~L} \cdot \mathrm{min}^{-1}$ observed following exposure to WBC compared to no change during $\Delta$ control. No differences between males and females were observed for $\dot{\mathrm{V}} \mathrm{E} \Delta$ control $(U=22, p=.178)$ or $\Delta \mathrm{WBC}(U=30, p=.834)$. A similar pattern was observed for $\dot{\mathrm{V}} \mathrm{O}_{2}$, for which $\triangle \mathrm{WBC}$ was significantly greater than $\Delta$ control $(Z=-3.516$, $p<.001$ ), with an increase of 0.4 L. $\mathrm{min}^{-1}$ observed following exposure to WBC compared to no change during $\Delta$ control. No differences between males and females were observed for $\dot{V}$ $\mathrm{O}_{2} \Delta$ control $(U=17, p=.068)$ or $\Delta \mathrm{WBC}(U=10, p=.021)$. For METS, $\Delta \mathrm{WBC}$ was significantly greater than $\Delta \operatorname{control}(Z=-3.516, p<.001)$, with an increase of 1.0 METS observed following exposure to WBC compared to 0.1 during $\Delta$ control. Interestingly, a significant difference was observed between males and females for METS $\Delta$ control $(U=11$, $p=.016$ ), where the mean change in METS from pre- to post-treadmill walking marginally increased in males (0.3) but decreased in females (-0.1). A similar trend was noted in METS $\triangle \mathrm{WBC}$, where males increased by 1.2 and females by 0.7 , however this was not statistically significant $(\mathrm{U}=11, p=.027)$.

\subsubsection{Cardiovascular measures}

No significant differences between conditions or sex were observed for diastolic blood pressure or HR. For systolic blood pressure, $\triangle \mathrm{WBC}$ was significantly greater than $\Delta$ control $(Z=-2.741, p=.006)$, with an increase of $3.0 \mathrm{mmHg}$ observed following exposure to WBC compared to a decrease of $9.1 \mathrm{mmHg}$ during $\Delta$ control. No differences between males and females were observed for systolic $\Delta$ control $(U=23, p=.210)$ or $\Delta \mathrm{WBC}(U=27, p=.597)$.

\subsubsection{Perceptual measures}

Descriptive data for thermal sensation are presented in Table 3. $\triangle \mathrm{WBC}$ was significantly greater than $\Delta$ control $(Z=-3.213, p=.001)$, with a decrease of 2 points on the TS scale observed following exposure to WBC compared to no change during $\Delta$ control. No differences between males and females were observed for TS $\Delta$ control $(U=33.5, p=.765)$, but females' TS decreased more than males following WBC, with females TS going from neutral to cold/very cold and males TS going from neutral to slightly cool. This difference was statistically significant $(U=2, p=.001)$. 
3.1.4 Exploring relationships between anthropometric measures and $\triangle W B C$ for ventilatory, metabolic and perceptual measures

For combined male and female data, a highly significant strong negative relationship was observed between fat mass index and $\dot{\mathrm{V}} \mathrm{O}_{2} \Delta \mathrm{WBC}(p=.002, r=-.718)$ and body fat percentage and $\dot{\mathrm{V}} \mathrm{O}_{2} \Delta \mathrm{WBC}$ ( $\left.p=.003, r=-.700\right)$. Similarly, a highly significant strong negative relationship was observed between fat mass index and METS $\triangle \mathrm{WBC}(p=.001, r=-.729)$ and body fat percentage and METS $\Delta \mathrm{WBC}(p=.004, r=-.676)$. Collectively, these data indicate that participants with greater adiposity demonstrated smaller changes in oxygen consumption and energy expenditure when exposed to WBC than leaner participants. $\triangle \mathrm{TS}$ (for which the negative changes observed indicates participants felt colder), demonstrated strong, statistically significant positive correlations with BSA $(p<.001, r=.776)$ and FFMI ( $p=.001 p=.001, r=.745)$, and a strong, statistically significant negative correlation with BSA to mass ratio $(p=.005, r=-.669)$. Collectively, these data indicate that smaller participants experience the cold more than larger participants, and leaner participants experience the cold more than less lean participants. No other significant associations were observed (Table 4). 
Table 3 Descriptive perceptual measures data

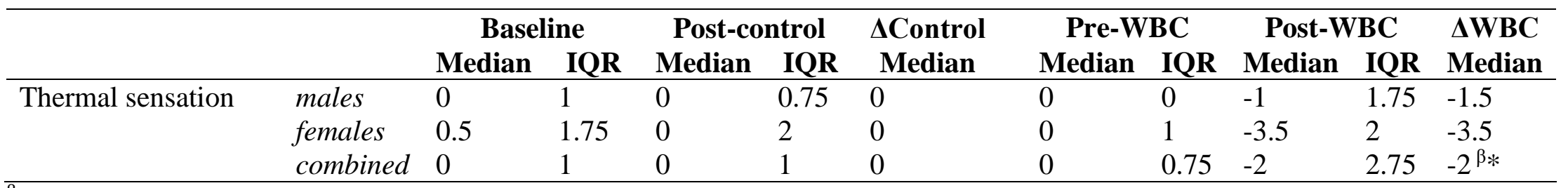

${ }^{\beta}$ statistically significant difference between males and females, $p \leq .001$

*statistically significant difference between $\Delta$ control and $\Delta \mathrm{WBC}, p \leq .001$

Table $4 r$ values and significance of correlations of anthropometric measures with $\triangle W B C$ for ventilatory, metabolic and perceptual measures

\begin{tabular}{|c|c|c|c|c|c|c|c|c|c|c|}
\hline & \multicolumn{2}{|c|}{$\dot{\mathrm{V}} \mathbf{E}\left(\right.$ L.min $\left.^{-1}\right)$} & \multicolumn{2}{|c|}{$\dot{\mathrm{V}} \mathbf{O}_{2}\left(\right.$ L.min $\left.\mathbf{m i n}^{-1}\right)$} & \multicolumn{2}{|c|}{ METS } & \multicolumn{2}{|c|}{ RER } & \multicolumn{2}{|c|}{ TS } \\
\hline & $r$ & $P$ & $\boldsymbol{r}$ & $\boldsymbol{P}$ & $r$ & $\boldsymbol{P}$ & $r$ & $\boldsymbol{P}$ & $r$ & $\boldsymbol{P}$ \\
\hline Body Mass Index $\left(\mathrm{kg} / \mathrm{m}^{2}\right)$ & -.104 & .700 & .028 & .918 & -.168 & .535 & .299 & .261 & .519 & .040 \\
\hline Body Surface Area $\left(\mathrm{m}^{2}\right)$ & .265 & .322 & .597 & .015 & .435 & .092 & -.132 & .625 & .776 & $.000 *$ \\
\hline BSA to Mass Ratio $\left(\mathrm{cm}^{2} / \mathrm{kg}\right)$ & -.068 & .803 & -.288 & .279 & -.091 & .737 & -.115 & .672 & -.669 & $.005^{*}$ \\
\hline Fat Mass Index $\left(\mathrm{kg} / \mathrm{m}^{2}\right)$ & -.194 & .471 & -.718 & $.002 *$ & -.729 & $.001 *$ & .532 & .034 & -.475 & .063 \\
\hline Fat Free Mass Index $\left(\mathrm{kg} / \mathrm{m}^{2}\right)$ & .044 & .871 & .459 & .074 & .326 & .217 & -.165 & .542 & .745 & $.001 *$ \\
\hline Body fat percentage (BIA) & -.171 & .528 & -.700 & $.003 *$ & -.676 & $.004 *$ & .506 & .046 & -.591 & .016 \\
\hline
\end{tabular}

*Statistically significant correlation, $p \leq .008$ 


\subsection{Phase 2 results}

Mean breathing frequency, expired volume, METS and oxygen consumption all increased immediately following exposure to WBC compared to 2 minutes of resting measures prior to WBC (see figure 1). These measures returned to baseline, or near baseline, during the second minute post-WBC.
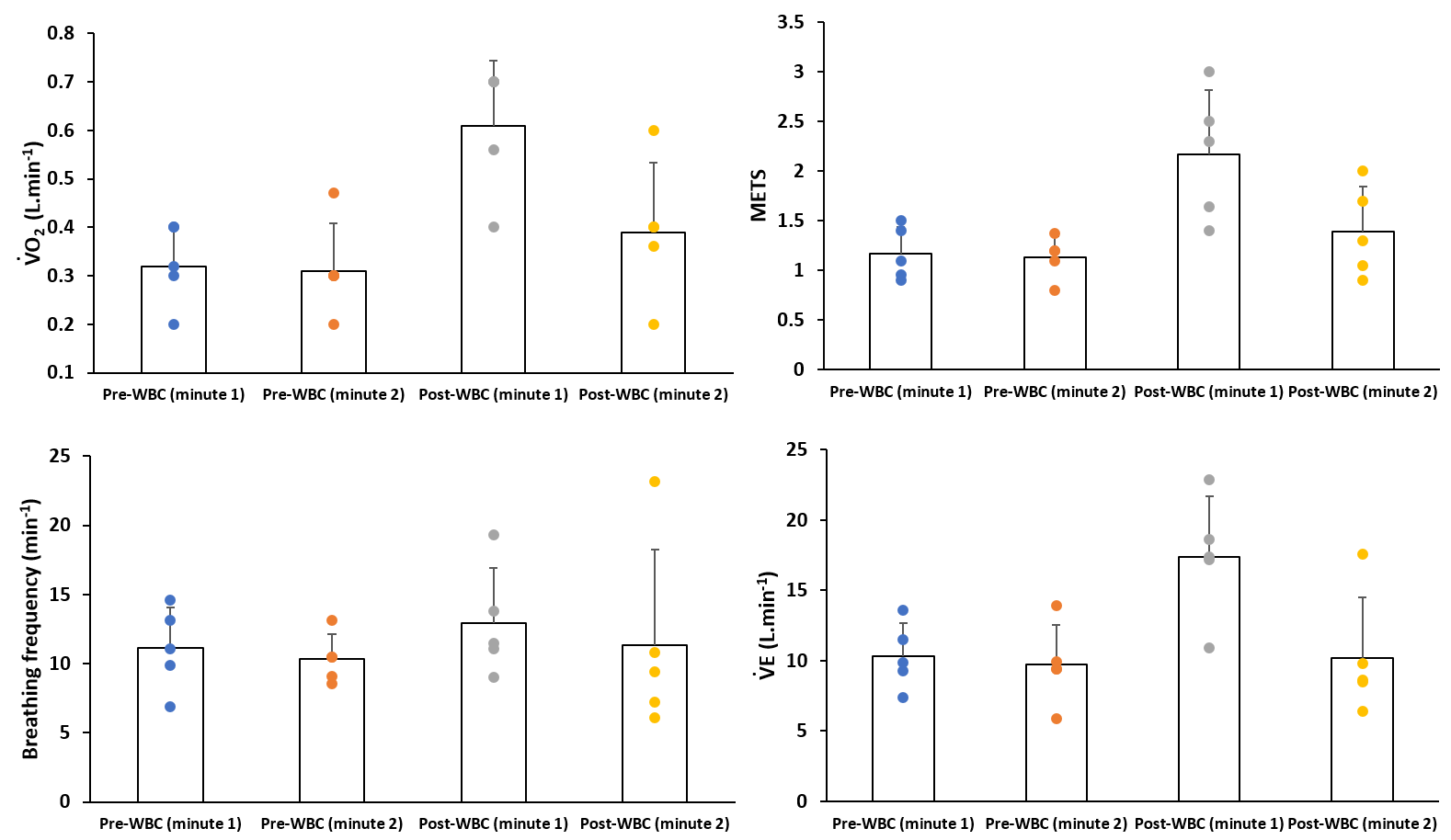

Figure 1 - combination graphs showing transient increases in mean respiratory measures post-WBC

\subsection{Discussion}

The primary aims of this study were to investigate respiratory responses and explore effects on energy expenditure after WBC, and to examine the role of sexual dimorphic anthropometric characteristics in relation to the shivering response and potential energy expenditure. Our study shows that a short exposure to WBC elicits a ventilatory response via increased expired volume, oxygen consumption and METS compared to a control condition. The largest differences occurred within the first minute post-exposure, but appear to return to near resting levels during the second minute post WBC, suggesting that the responses observed are transient. The increases in $\dot{\mathrm{V}} \mathrm{O}_{2}, \dot{\mathrm{V}} \mathrm{E}$ and METS observed post WBC are likely to be due to an acute transient shivering response due to the cold air exposure. Under cold conditions, the body adjusts to maintain heat balance, which can be through heat conservation, via vasoconstriction and the insulative response, or through heat production, 
that is non-shivering or shivering thermogenesis. Shivering thermogenesis is one of the ways in which the body creates heat via the muscle shivering or creating rapid movement, to adapt or respond to the environmental stress. Exposure to WBC and the concomitant effects of a very rapid decrease in skin temperature may have a profound enough effect to elicit a ventilatory and potentially shivering response. However, making a definitive link between WBC and shivering thermogenesis is difficult from these findings, as we did not measure core or skin temperature, and did not systematically seek to observe the presence of shivering.

No notable differences in change in respiratory responses were observed between sexes. This is despite the differences in male and female physiology, i.e. larger chest cavity and lung volume in males compared to females. For $\dot{\mathrm{V}} \mathrm{E}, \dot{\mathrm{V}} \mathrm{O}_{2}$ and METS, females demonstrated smaller values than males (for example, resting $\dot{V} \mathrm{E}$ was $16.2 \mathrm{~L} \cdot \mathrm{min}^{-1}$ in males compared to 10.1 L.min ${ }^{-1}$ in females) however the magnitude of change $(\Delta)$ for these parameters was very similar between sexes ( $\dot{V}$ E increased by $12.1 \mathrm{~L}^{\mathrm{min}}{ }^{-1}$ in males and 9.4 L.min ${ }^{-1}$ in females). This suggests that sex and sexual dimorphism is not a factor in ventilatory and metabolic responses to WBC. However, adiposity was associated with $\dot{\mathrm{V}}_{2} \triangle \mathrm{WBC}$ and METS $\triangle \mathrm{WBC}$, with fatter participants demonstrating smaller changes in oxygen consumption and energy expenditure when exposed to WBC than leaner participants. These findings suggest that the skinnier the participant the greater their respiratory response, potentially via metabolic and shivering thermogenesis to maintain core temperature. Previous work comparing cooling between sexes following WBC indicated skin temperature in females cooled more quickly (Hammond et al., 2014; Cuttell et al., 2017) and that women had a greater insulative capacity to withstand the cold compared to males. This clear sex difference was not borne out in these data, however the associated differences in adiposity were. As a consequence, those individuals that had less body fat may have compensated for the heat loss by shivering (albeit acutely), thereby demonstrating an increased ventilatory response. Alternatively, as cold environmental stress is often associated with increases in ventilatory responses (gasp, hyperventilation) (Tipton, 1989), it is also possible that the participants experienced an acute transient cold shock response. It is also possible that differences in the testing protocol between males and females contributed to the lack of significant sex differences observed, as control data were collected in the morning for female participants and in the afternoon for male participants. There is evidence of a role of circadian timing on regulation of breathing, 
with circadian rhythms observed in oral temperature and respiratory chemoreflex responses, but not in metabolic rate in healthy male participants (Stephenson et al., 2000). Further research in this area is required in order to fully understand the phenomena observed.

Previous studies in the fields of WBC (Cuttell et al., 2017, Bouzigon et al., 2018) and coldwater immersion (McArdle et al., 1984; Glickman-Weiss et al., 2000) have demonstrated that males and females may potentially experience exposure to extreme cold differently. The current investigation observed females to report thermal sensation immediately after WBC of around -3.5 (cold to very cold) while males reported -1 (slightly cool), with the decrease in TS being significantly greater in females that in males. The findings show that smaller participants experience the cold more than larger participants, and leaner participants experience the cold more than less lean participants, which corroborates the findings of Bouzigon et al. (2018) who noted that "special attention should be given to female athletes with a low BMI as they seem to be much more sensitive to cold" (pp. 60). However, whether the perception of cold has any link with the autonomic effect of breathing and ventilation following WBC has yet to be established. Instead these data should be considered with interest given the subjective differences experienced between the sexes and indeed an anticipatory acute ventilatory response difference following WBC. The participants were not naive to the hypothesis and therefore may have engendered a certain response for the investigators. Therefore, it would be interesting for future research to address a placebocontrolled design investigating different temperatures and ventilatory responses (i.e. -40 versus -80 versus $-110^{\circ} \mathrm{C}$ ), with participants blinded or deceived as to the actual temperature, in an attempt to control for this limitation in the current study. A further limitation of this study is that it is possible that order effects could have affected these data as the order of exposure to the two conditions was not randomised, with all participants completing the control condition first and WBC condition second. Adopting a randomised cross over design in future would mitigate against this potential risk of bias. The different timing in control testing protocol between males and females (afternoon versus morning respectively) and the potential for circadian rhythm to affect the comparability of these data, as already mentioned, is a limitation of this study. Furthermore, some of the differences noted for male participants, for example systolic blood pressure, could have been influenced by the control and WBC data collection taking place on different days one month apart. 
To the best of our knowledge, this is the first study to consider ventilatory and metabolic effects of WBC. Previous studies have explored the effects of repeated exposures on lung function (Smolander et al., 2006), lipid profile and oxidative stress (Lubkowska et al., 2010; Stanek et al., 2016; Stanek et al., 2019) and body composition (Filliard et al., 2016) and have shown a variety of different data. As a result some commercial manufacturers have claimed that frequent cold exposure can increase fat metabolism and therefore fat burn, yet realistically multiple chronic-based exposures are required in order to see any effects (Lubkowska et al., 2010; Filliard et al., 2016). Our study demonstrated that a single, acute cold exposure increases respiratory markers, albeit in a transient way, that does not support commercial claims of fat burning. However, these findings support a growing body of evidence in WBC that indicates that responses to WBC differ according to anthropometry, and practitioners or organisations delivering WBC require a greater understanding of the optimised dose required for participants based on their sex and body mass.

\subsection{Methodological considerations}

The secondary aim of this study was to explore the feasibility of collecting metabolic data following WBC using different methods. In the main study, phase 1, Douglas bags were used to collect ventilatory data. In phase 2 , breath by breath analysis was piloted. There are clear limitations to Phase 2 of this study, which was exploratory in nature and involved ventilatory measures being taken from just five participants with a Cortex metalyser. Because of the very small numbers involved, no inferential statistics were performed with these data, and some caution should be exercised when interpreting the findings presented. However, this phase of the study has provided some additional insight into the very acute nature of the ventilatory changes observed, and provides a platform for future investigation in this area. However, limiting both phases of the study is that these findings describe only the ventilatory and metabolic status of participants before and after exposure to WBC; currently, we do not know the ventilatory or metabolic responses during WBC itself. Future research should be directed towards finding a method that can withstand the extreme temperatures of a WBC chamber in order to measure these parameters during the exposure itself.

\subsection{Conclusion}

This is the first study to investigate the respiratory responses to $\mathrm{WBC}$ and we found a difference between following WBC, that varied by anthropometry. There seems to be no set protocol for WBC use and a one size fits all approach could potentially impact on the 
participants using the treatment over a chronic time period. For example, it has been previously shown that cross county skiers have a prevalence to develop asthma symptoms, bronchial hypersensitivity and even increase inflammatory cell (granulocytes and macrophages) responses in the lungs, with the author suggesting that long term repeated exposure to the cold may induce asthma (Larsson et al., 1998). Thus, practioners and users of WBC need to remain aware of all issues and the potential limitations of use over time of such a cold treatment. Our data showed an increase in ventilatory responses in healthy participants and further, given that some athletes are repeatedly exposed to $\mathrm{WBC}$, the consequences of such a treatment are not yet known.

\section{Acknowledgements}

The authors would like to thank Dr Joe Costello and Mr Dominic Langdon for support with data collection and the participants who volunteered to take part in this study.

\section{References}

Bouzigon, R., Ravier, G., Dugue, B. and Grappe, F. (2018) Thermal Sensations during a Partial-Body Cryostimulation Exposure in Elite Basketball Players. Journal of Human Kinetics. 62. 55-63. DOI: 10.1515/hukin-2017-0158

Cholewka, A., Stanek, A., Sieroń, A. and Drzazga, Z. (2012) Thermography study of skin response due to whole-body cryotherapy. Skin Research and Technology. 18(2). 180-187. DOI: $10.1111 / \mathrm{j} .1600-0846.2011 .00550$

Costello, J.T., Baker, P.R., Minett, G.M., Bieuzen, F., Stewart, I.B. and Bleakley, C. (2015) Whole-body cryotherapy (extreme cold air exposure) for preventing and treating muscle soreness after exercise in adults (Review). Cochrane Database of Systematic Reviews. 9, CD010789. DOI: 10.1002/14651858.CD010789.pub2.

Cuttell, S., Hammond, L., Langdon, D. and Costello, J. (2017) Individualising the exposure of $-110^{\circ} \mathrm{C}$ whole body cryotherapy: The effects of sex and body composition. Journal of Thermal Biology. 65. 41-47. DOI: 10.1016/j.jtherbio.2017.01.014

Datta, A. and Tipton, M. (2006) Respiratory responses to cold water immersion: neural pathways, interactions, and clinical consequences awake and asleep. Journal of Applied Physiology. 100(6). 2057-2064. DOI: 10.1152/japplphysiol.01201.2005

DuBois, D. and DuBois, E. F. (1916) Clinical Caliometry. Tenth Paper. A formula to estimate the approximate surface area if height and weight be known. Archives of Internal Medicine. 17. 863-871. DOI: 10.1001/archinte.1916.00080130010002. 
Engel, P., Fricke, R., Taghawinejad, M. and Hildebrandt, G. (1989) Lungenfunktion und Ganzkörperkältebehandlung bei Patienten mit chronischer Polyarthritis. Z Phys Med Baln Med Klim. 18. 37-43.

Filliard, J., Faria, F.C., Bieuzen, F., Berthelot, G. and Volondat, M. (2016) The effects of the whole body cryotherapy on the body composition. British Journal of Sports Medicine. 50:A38. DOI: 10.1136/bjsports-2016-097120.65.

Glickman-Weiss, E.L., Cheatham, C., Caine, N., Blegen, M., Marcinkiewicz, J. and Mittleman, K.D. (2000) The influence of gender and menstrual phase on thermosensitivity during cold water immersion. Aviation, Space, and Environmental Medicine. 71(7). 715-722.

Hammond, L. E., Cuttell, S., Nunley, P. and Meyler, J. (2014). Anthropometric characteristics and sex influence magnitude of skin cooling following exposure to whole body cryotherapy. BioMed Research International. 628724. DOI: 10.1155/2014/628724.

Larsson, K., Tornling, G., Gavhed, D., Müller-Suur, C. and Palmberg, L. (1998) Inhalation of cold air increases the number of inflammatory cells in the lungs in healthy subjects. European Respiratory Journal. 12(4). 825-830.

Lombardi, G., Ziemann, E. and Banfi, G. (2017) Whole-Body Cryotherapy in Athletes: From Therapy to Stimulation. An Updated Review of the Literature. Frontiers in Physiology. 2(8). 258. DOI: 10.3389/fphys.2017.00258.

Lubowska, A., Banfi, G., Dolegowska, B., Melzi D’Eril, G., Luczak, J. and Barassi, A. (2010) Changes in lipid profile in response to three different protocols of whole-body cryostimulation treatments. Cryobiology. 61. 22-26. DOI: 10.1016/j.cryobiol.2010.03.010.

McArdle, W.D., Magel, J.R., Gergley, T.J., Spina, R.J. and Toner, M.M. (1984). Thermal adjustment to cold-water exposure in resting men and women. Journal of Applied Physiology. 56. 1565-1571. DOI: 10.1152/jappl.1984.56.6.1565.

Rymaszewska, J., Ramsey, D. and Chladzinska-Kiejna, S. (2008) Whole-body cryotherapy as adjunct treatment of depressive and anxiety disorders. Archivum Immunologiae et Therapiae Experimentalis. 56. 63-68. DOI: 10.1007/s00005-008-0006-5.

Selfe, J., Alexander, J., Costello, J. T., May, K., Garratt, N., Atkins, S., Dillon, S., Hurst, H., Davison, M., Przybyla, D., Coley, A., Bitcon, M., Littler, G. and Richards, J. (2014) The effect of three different $\left(-135^{\circ} \mathrm{C}\right)$ whole body cryotherapy exposure durations on elite rugby league players. PLoS ONE. 9(1). e86420. DOI: 10.1371/journal.pone.0086420.

Smolander, J., Westerlund, T., Uusitalo, A., Dugué, B., Oksa, J. and Mikkelsson, M. (2006) Lung function after acute and repeated exposures to extremely cold air $\left(-100^{\circ} \mathrm{C}\right)$ during whole-body cryotherapy. Clinical Physiology and Functional Imaging. 26(4). 232-234.

Stanek, A., Cholewka, A., Wielkoszyński, T., Romuk, E., and Sieroń, A. (2018) Whole-body cryotherapy decreases the levels of inflammatory, oxidative stress, and atherosclerosis plaque markers in male patients with active-phase ankylosing spondylitis in the absence of classical 
cardiovascular risk factors. Mediators of Inflammation. 8592532.

DOI: $10.1155 / 2018 / 8592532$.

Stanek, A., Romuk, E., Wielkoszynski, T., Bartus, S., Cieślar, G. and Cholewka, A. (2019) Decreased lipid profile and oxidative stress in healthy subjects who underwent whole-body cryotherapy in closed cryochamber with subsequent kinesiotherapy. Oxidative Medicine and Cellular Longevity. 7524878. DOI: 10.1155/2019/7524878.

Stanek, A., Sieroń-Stoltny, K., Romuk, E., Cholewka, A., Wielkoszyński, T., Cieślar, G., Kwiatek, S., Sieroń, A. and Kawczyk-Krupka, A. (2016) Whole-body cryostimulation as an effective measure of reducing oxidative stress in healthy men. Advances in Clinical and Experimental Medicine. 25(6). 1281-1291. DOI: 10.17219/acem/65980.

Stephenson, R., Mohan, R.M., Duffin, J. and Jarsky, T.M. (2000) Circadian rhythms in the chemoreflex control of breathing. American Journal of Physiology-Regulatory, Integrative and Comparative Physiology. 278(1). R282-R286.

Tipton, M.J. (1989) The initial responses to cold-water immersion in man. Clinical Science. 77. 581-588.

Tipton, M. J., Franks, G. M., Meneilly, G. S. and Mekjavic, I. B. (1997) Substrate utilisation during exercise and shivering. European Journal of Applied Physiology and Occupational Physiology. 76. 103-108.

Vallerand, A.L. and Jacobs, I. (1989) Rates of energy substrates utilization during human cold exposure. European Journal of Applied Physiology and Occupational Physiology. 58. 873-878. DOI: 10.1007/BF02332221.

Westerlund, T., Oksa, J., Smolander, J. and Mikkelsson, M. (2003) Thermal responses during and after whole-body cryotherapy $\left(-110^{\circ} \mathrm{C}\right)$. Journal of Thermal Biology. 28. 601-608.

Yamauchi, T. (1986) Whole-body cryotherapy is a method of extreme cold $\left(-175{ }^{\circ} \mathrm{C}\right)$ treatment initially used for rheumatoid arthritis. Z Phys Med Baln Med Klim. 15. 311. 